\title{
Recombinant human soluble thrombomodulin protects against brain injury in a CVST rat model, via downregulation of the HMGB1-RAGE axis
}

\author{
JIAN-JUN GU ${ }^{1 *}$, JIE-BIN CHEN ${ }^{2 *}$, JIAN-HE ZHANG ${ }^{1}$, HAO ZHANG $^{1}$ and SHOU-SEN WANG ${ }^{1}$ \\ ${ }^{1}$ Department of Neurosurgery, Fuzhou General Hospital, Xiamen University Medical College, Fuzhou, Fujian 350025; \\ ${ }^{2}$ Department of Pediatrics, Taizhou People's Hospital Affiliated to the Medical College of Nantong University, \\ Taizhou, Jiangsu 225300, P.R. China
}

Received April 21, 2016; Accepted July 27, 2016

DOI: $10.3892 / \mathrm{mmr} .2016 .5891$

\begin{abstract}
Cerebral venous sinus thrombosis (CVST) is a distinct cerebrovascular disorder, and $\sim 50 \%$ of CVST patients progress to cerebral venous infarction, resulting in elevation of cerebral venous pressure. Anticoagulation is the standard initial treatment and is associated with a reduced relative risk of mortality and dependency. Recombinant human soluble thrombomodulin (rhs-TM) is a promising therapeutic natural anticoagulant comparable to antithrombin, tissue factor pathway inhibitor, and activated protein $\mathrm{C}$. The present study aimed to investigate the protective effects of rhs-TM in a CVST rat model, and identify any underlying mechanisms. Rats were treated with rhs-TM intravenously prior to CVST. Following neurological function evaluation, animals were sacrificed and brain water content and infarct volume were assessed. Brain tissue was collected from the infarcted segments and mRNA and protein expression levels of high mobility group box 1 (HMGB1), receptor for advanced glycation end products (RAGE), tumor necrosis factor (TNF)- $\alpha$, interleukin (IL)-1 $\beta$, IL-6, caspase-3, B-cell lymphoma-2 and Bcl-2 associated X were analyzed by reverse transcription-quantitative polymerase chain reaction and western blot analysis. rhs-TM significantly prevented neurological deficits in locomotor function and reduced infarct volume. The expression levels of HMGB1-RAGE were upregulated in the infarcted segments of rat brains following CVST. Pretreatment with rhs-TM
\end{abstract}

Correspondence to: Dr Shou-Sen Wang, Department of Neurosurgery, Fuzhou General Hospital, Xiamen University Medical College, 156 Xi'erhuanbei Road, Fuzhou, Fujian 350025, P.R. China

E-mail:wshsen@xmu.edu.cn

*Contributed equally

Key words: cerebral venous sinus thrombosis, recombinant human soluble thrombomodulin, HMGB1-RAGE axis, apoptosis, inflammation cytokines inhibited the HMGB1-RAGE axis, alleviating the expression levels of the proinflammatory cytokines, TNF- $\alpha$, IL-1 $\beta$ and IL-6; however, expression levels of the apoptosis-associated genes and proteins remained unaffected. The results of the present study indicated that rhs-TM protects against CVST in the rat model via inhibition of the HMGB1-RAGE axis and inflammation, but not via apoptosis.

\section{Introduction}

Cerebral venous sinus thrombosis (CVST) is a rare cerebrovascular disorder representing between 0.5 and $3 \%$ of all strokes, which predominantly affects younger people $(1,2)$. Patients with CVST develop venous infarcts in $\sim 50 \%$ of cases, often resulting in a spectrum of clinical manifestations, including headache, hemiparesis, seizures and intracranial hypertension with papilledema (3). Although modern imaging techniques, in combination with improved treatment of CVST, have significantly improved survival and clinical outcomes in recent decades, the pathogenesis of CVST remains poorly understood and effective treatment strategies remain to be elucidated. The use of heparin and oral anticoagulants is based on the rationale of reversing the causal thrombotic process and preventing complications. However, due to the presence of a hemorrhagic element in $40 \%$ of CSVT cases, the administration of anticoagulant treatment remains controversial (4). One previous study suggested that early anticoagulation may be beneficial, although it was associated with an increased risk of symptomatic brain hemorrhage (5). Therefore, a safer alternative therapy is required to provide marked benefits to CVST patients.

Thrombomodulin (TM) is a glycoprotein present in the membranes of endothelial cells, which regulates coagulation via effects on thrombin. TM acts as a cofactor for the thrombin-catalyzed activation of protein $\mathrm{C}$, enhancing the reaction rate $>1,000$-fold, switching thrombin from a procoagulant to an anticoagulant enzyme $(6,7)$, and exerts anti-inflammatory effects (8). A previous study demonstrated that Solulin, a recombinant soluble analog of human $\mathrm{TM}$, facilitated recanalization and reduced stroke volume following middle cerebral artery occlusion (9), and decreased 
the expression levels of the proinflammatory cytokines, tumor necrosis factor (TNF)- $\alpha$, interleukin (IL)-1 $\beta$ and IL-6 in the infarct, compared with control animals (10). These studies revealed a protective effect of Solulin against ischemic brain damage, suggesting that soluble TM exerts anticoagulant and anti-inflammatory effects following stroke. However, whether recombinant human soluble (rhs)-TM has a neuroprotective effect in the CVST model remains to be elucidated.

In the present study, the expression levels of proinflammatory cytokines and apoptosis genes in the infarcted segments of CVST rat brains were analyzed following rhs-TM treatment, to assess its neuroprotective effect and reveal any underlying molecular mechanisms.

\section{Materials and methods}

Animal preparation. The present study was approved by the ethics committee of Fuzhou General Hospital (Fuzhou, China), and all animal experiments were performed in accordance with the institutional guidelines on the care and use of experimental animals. A total of 54 male Sprague-Dawley rats (weight, 240-260 g; age, 6 weeks old), obtained from the Shanghai SLAC Laboratory Animal Co., Ltd. (Shanghai, China), were used in the present study. Rats were housed in standard conditions at $26-28^{\circ} \mathrm{C}$, with $10 \mathrm{~h}$ light $/ 14 \mathrm{~h}$ dim light cycles and access to food and water ad libitum. Of the 54 rats, 36 were randomly assigned to six subgroups ( $\mathrm{n}=6$ rats/group) and one group was sacrificed by intraperitoneal injection of $30 \mathrm{mg} / 100 \mathrm{~g}$ body weight chloral hydrate at 0, 6, 12, 24, 48 and $72 \mathrm{~h}$ following the induction of thrombus. The remaining 18 rats were divided into three groups ( $\mathrm{n}=6$ rats/group): Sham, CVST, and rhs-TM. The rhs-TM group was injected with $1 \mathrm{mg} / \mathrm{kg}$ rhs-TM (dissolved in saline; Asahi Kasei Pharma Corp., Tokyo, Japan) through the tail vein $10 \mathrm{~min}$ prior to CVST. The CVST and the sham groups received normal saline.

Surgical preparation and CVST model. In all experiment groups, rats were anesthetized with an intraperitoneal injection $30 \mathrm{mg} / 100 \mathrm{~g}$ body weight chloral hydrate. To induce venous sinus thrombosis, a $1.5 \times 10 \mathrm{~mm}$ cranial window was made to expose the superior sagittal sinus. A strip of filter paper soaked with $40 \%$ ferric chloride was applied to the exposed cranial window for $5 \mathrm{~min}$, whereas the sham group received filter paper soaked with $0.9 \%$ saline. Subsequently, the ferric chloride strip was removed and the field flushed with $0.9 \%$ saline; the removed bone strip was replaced, sealed with bone cement and the skin sutured.

Neurological evaluation. Neurological evaluations were performed in a blinded manner. Rats were subjected to neurological evaluation prior to surgery and at $24 \mathrm{~h}$ following CVST using a score of $0-3$, where $0=$ no observable neurological deficit (normal), $1=$ failure to extend left forepaw on lifting whole body by tail (mild), $2=$ circling to contralateral side (moderate) and 3=no spontaneous motor activity (severe) (11).

\section{2,3,5-triphenyltetrazolium chloride (TTC) staining and stroke} volume. Rats were sacrificed with an intraperitoneal injection of $30 \mathrm{mg} / 100 \mathrm{~g}$ body weight chloral hydrate $24 \mathrm{~h}$ following CVST. Brains were removed, cut into 2-mm-thick coronal sections, stained with $1 \%$ TTC in phosphate-buffered saline for $20 \mathrm{~min}$ at $37^{\circ} \mathrm{C}$ and fixed in $10 \%$ paraformaldehyde solution for $10 \mathrm{~min}$. The sections were analyzed with the image analysis software Image J version 1.44 (National Institutes of Health, Bethesda, MD, USA). For all 6 slices, the brain infarct volume was calculated as the product of the average slice thickness (2-mm) and the sum of the infarction area.

Brain water content. The water content of the brain was determined by the dry-wet weight method. The brains were removed following sacrifice by chloral hydrate administration and the cerebellum, pons and olfactory bulbs from each brain were removed. Each cerebrum was weighed to determine its wet weight. The brains were subsequently placed into an $110^{\circ} \mathrm{C}$ thermal oven for $24 \mathrm{~h}$, and weighed to determine their dry weight. Water content was calculated using the following formula: Water content $=[$ (wet weight - dry weight $) /$ wet weight] x 100 .

Reverse transcription-quantitative polymerase chain reaction $(R T-q P C R)$. Total RNA was isolated from the penumbra regions of the infarcted hemispheres using TRIzol ${ }^{\circledR}$ reagent (Invitrogen; Thermo Fisher Scientific, Inc. Waltham, MA, USA), according to the manufacturer's instructions. cDNA was generated with the High-Capacity cDNA RT kit (Roche Diagnostics GmbH, Mannheim, Germany). The mRNA expression levels of high mobility group box 1 (HMGB1), receptor for advanced glycation end products (RAGE), TNF- $\alpha$, IL-1 $\beta$, IL- 6 , caspase-3, B-cell lymphoma-2 (Bcl-2) and Bcl-2 associated X (Bax) were analyzed by qPCR using a SYBR Green PCR Master Mix kit (Applied Biosystems; Thermo Fisher Scientific, Inc.) in conjunction with an ABI-Prism 7300 system (Applied Biosystems; Thermo Fisher Scientific, Inc.). Thermal cycling parameters consisted of an initial enzyme activation step at $95^{\circ} \mathrm{C}$ for $10 \mathrm{~min}$, followed by 40 cycles of denaturation at $95^{\circ} \mathrm{C}$ for $15 \mathrm{sec}$, annealing at $60^{\circ} \mathrm{C}$ for $30 \mathrm{sec}$ and amplification at $72^{\circ} \mathrm{C}$ for $30 \mathrm{sec}$. The concentrations of the target genes were calculated by comparing quantification cycle $\left(\mathrm{C}_{\mathrm{q}}\right)$ values in each sample with $\mathrm{C}_{\mathrm{q}}$ values of the internal standard curve (12). Melting curve analysis and gel electrophoresis evaluation of RT-qPCR products was routinely performed to determine the specificity of the reaction. The mRNA expressions levels were normalized to glyceraldehyde 3-phosphate dehydrogenase (GAPDH) mRNA levels. The following primers were used: Forward, 5'-CGAGTC CGTGTCTACCAGATT-3' and reverse, 5'-GCGGCTGGA ATGGAAACTGAA-3' for RAGE; forward, 5'-GCTCCA TAGAGACAGCGCCGGG-3' and reverse, 5'-CCTCAGCGA GGCACAGAGTCGC-3' for HMGB1; forward, 5'-CTCCCA GAAAAGCAAGCAAC-3' and reverse, 5'-CGAGCAGGA ATGAGAAGAGG-3' for TNF- $\alpha$; forward, 5'-CTGTGACTC GTGGGATGATG-3' and reverse, 5'-GGGATTTTGTCG TTGCTTGT-3' for IL-1 $\beta$; forward, 5'-ACAGTGCATCAT CGCTGTTC-3' and reverse, 5'-CCGGAGAGGAGACTT CACAG-3' for IL-6; forward, 5'-CTGGACTGCGGTATT GAG-3' and reverse, 5'-GGAACATCGGATTTGATT-3' for caspase-3; forward, 5'-ATACCTGGGCCACAAGTGAG-3' and reverse, 5'-TGATTTGACCATTTGCCTGA-3' for Bcl-2; forward, 5'-CGAGCTGATCAGACATCA-3' and reverse, 
5'-CTCAGCCCATCTTCTTCCAG-3' for Bax; and forward, 5'-TCGGAGTCAACGGATTTGG-3' and reverse, 5'-CAT GGTGGATCATGGA-3' for GAPDH.

Western blot analysis. Rats were sacrificed with an intraperitoneal injection of $30 \mathrm{mg} / 100 \mathrm{~g}$ body weight chloral hydrate and penumbra regions of the infarcted hemispheres were lysed in radioimmunoprecipitation assay buffer (EMD Millipore, Billerica, MA, USA). The concentration of the protein extracts was determined by the Bradford assay (Bradford Protein assay kit; Bio-Rad Laboratories, Inc., Hercules, CA, USA). A total of $40 \mu \mathrm{g}$ protein was loaded onto gels, separated by $8-15 \%$ SDS-PAGE and transferred onto polyvinylidene difluoride membranes. Membranes were blocked with $5 \%$ non-fat milk in Tris-buffered saline containing $0.1 \%$ Tween-20, and subsequently probed with primary antibodies overnight at $4^{\circ} \mathrm{C}$. The following antibodies were used: Monoclonal rabbit anti-HMGB1 (cat. no. ab92310; dilution, 1:1,000; Abcam, Cambridge, MA, USA), monoclonal rabbit anti-RAGE (cat. no. ab172473; dilution, 1:500; Abcam), polyclonal rabbit anti-TNF- $\alpha$ (cat. no. ab6671; dilution, 1:500; Abcam), polyclonal rabbit anti-IL-1 $\beta$ (cat. no. ab9722; dilution, 1:500; Abcam), monoclonal mouse anti-IL-6 (cat. no. ab9324; dilution, 1:500; Abcam), polyclonal rabbit anti-caspase-3 (cat. no. ab4051; dilution, 1:500; Abcam), polyclonal rabbit anti-Bcl-2 (cat. no. ab59348; dilution, 1:500; Abcam) and polyclonal rabbit anti-Bax (cat. no. ab59348; dilution, 1:500; Abcam). The membranes were washed three times with PBS-Tween 20, before they were incubated with horseradish peroxidase-conjugated rabbit anti-mouse or goat anti-rabbit IgG secondary antibodies (H+L; cat. nos. ab6728 and ab6721, respectively; dilution, 1:2,000; Abcam) for $1 \mathrm{~h}$ at room temperature. The blots were developed using the SuperSignal ${ }^{\mathrm{TM}}$ West Pico Chemiluminescent Substrate (cat. no. 34077; Invitrogen; Thermo Fisher Scientific, Inc.). Band intensities were normalized to $\beta$-actin and measured using spot densitometry analysis with Image J software (version 1.44, National Institutes of Health).

Statistical analysis. Data are expressed as the mean \pm standard deviation. Statistical analyses were performed in SPSS software version 13.0 (SPSS, Inc., Chicago, IL, USA). Intergroup differences were analyzed using paired Student's $t$-test and multiple comparisons among various groups were conducted by analyses of variance with a post-hoc Tukey test. $\mathrm{P}<0.05$ was considered to indicate a statistically significant difference.

\section{Results}

Expression levels of HMGB1-RAGE increased in infarcted segments of CVST rat brains. The mRNA and protein expression levels of HMGB1-RAGE in the infarcted segments of CVST rat brains were analyzed by RT-qPCR and western blot analysis, respectively. HMGB1 mRNA expression levels were upregulated in the infarcted segments of rat brains $6 \mathrm{~h}$ following CVST, and RAGE mRNA expression levels were upregulated $12 \mathrm{~h}$ following CVST. mRNA expression levels of the two peaked at $24 \mathrm{~h}$ and gradually declined, although significant differences compared with the control group were observed even at $72 \mathrm{~h}$ following CVST $(\mathrm{P}<0.001$; Fig. 1A). Western blot analysis demonstrated that HMGB1 and RAGE protein
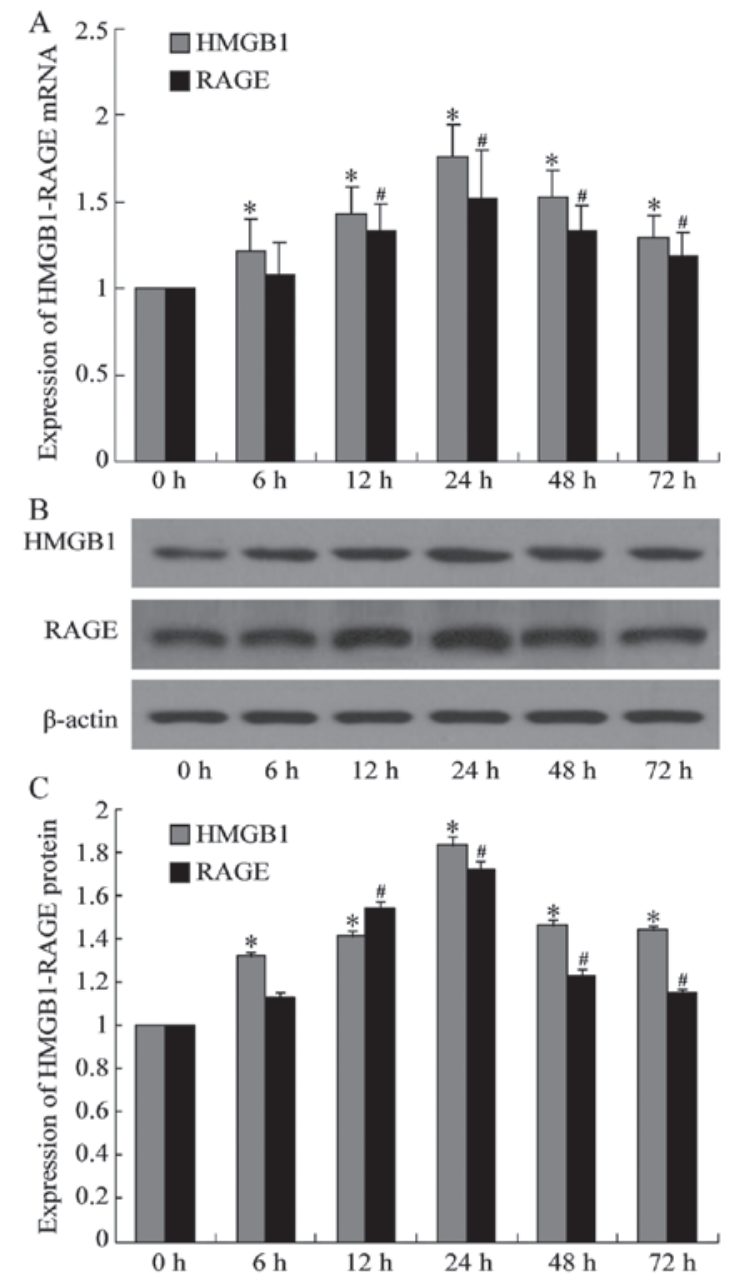

Figure 1. HMGB1 and RAGE mRNA and protein expression levels following CVST. (A) Reverse transcription-quantitative polymerase chain reaction analysis revealed that HMGB1 and RAGE mRNA expression levels were upregulated in the infarcted segments of the brains of rats that had undergone CVST. (B) Western blotting analysis and (C) quantification of western blots demonstrated that HMGB1 and RAGE protein expression levels were upregulated in the infarcted segments of the brains of rats that had undergone CVST. Data are expressed as the mean \pm standard deviation. ${ }^{*} \mathrm{P}<0.05$ vs. 0 h. HMGB1; ${ }^{\#} \mathrm{P}<0.05$ vs. 0 h. RAGE. HMGB1, high mobility group box 1 ; RAGE, receptor for advanced glycation endproducts; CVST, cerebral venous sinus thrombosis.

expression levels were consistent with the RT-qPCR data (Fig. 1B and C). The time point of $24 \mathrm{~h}$ was therefore selected for subsequent analyses.

rhs-TM may protect against neurological deficits. In the sham group no neurological deficits were observed. The neurological deficit score $24 \mathrm{~h}$ following surgery was $1.49 \pm 0.38$ in the CVST group and $1.02 \pm 0.29$ in the rhs-TM group ( $\mathrm{P}=0.036$; Fig. $2 \mathrm{~A})$. This suggests that rhs-TM may protect against neurological deficits.

rhs-TM reduced infarction volume. Brain infarctions were identified by TTC staining of the brain slices, revealing a bilateral infarction in the CVST group (Fig. 2B). The brain infarction volume was $83.3 \pm 4.6 \mathrm{~mm}^{3} 24 \mathrm{~h}$ following CVST alone, compared with $65.3 \pm 3.8 \mathrm{~mm}^{3}$ in the rhs-TM group $(\mathrm{P}<0.001$; Fig. 2C). No brain lesions were observed in the sham group. 
A
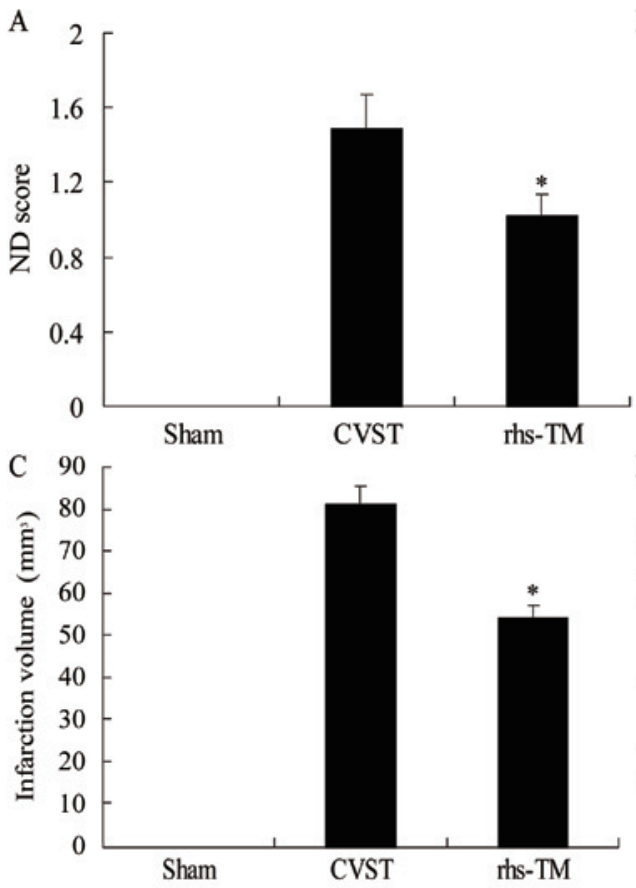

B

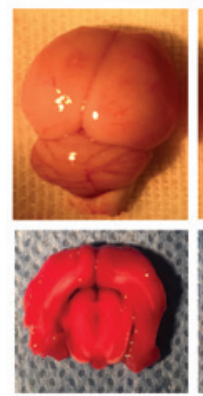

Sham

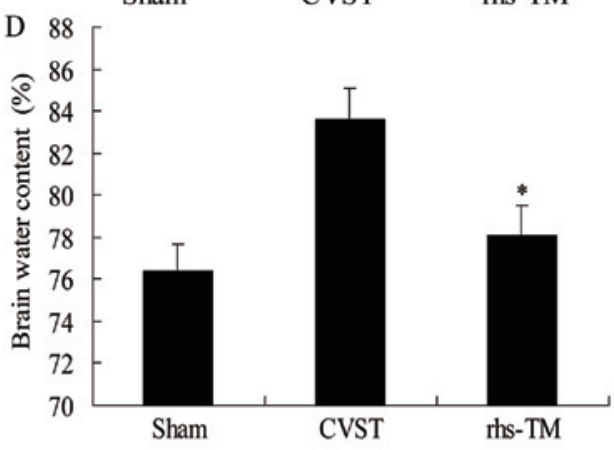

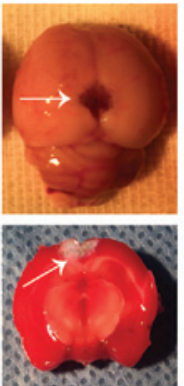

CVST

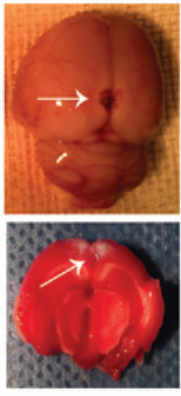

rhs-TM

Figure 2. Effect of rhs-TM on ND score, infarction volume and brain water content following CVST. (A) ND score was decreased in the rhs-TM group compared with the CVST alone group, $24 \mathrm{~h}$ following surgery. Sham group animals had no observable neurological deficits. (B) Photographs (magnification, $\mathrm{x} 100$ ) of unstained (top panel) and TTC-stained rat brains (bottom panel). Arrows indicate infarcted regions. (C) Quantification of infarction volume from TTC staining demonstrated that the infarction volume was decreased in the rhs-TM group compared with the CVST alone group, $24 \mathrm{~h}$ following surgery. (D) Brain water content was decreased in the rhs-TM group compared with the CVST alone group. Data are expressed as the mean \pm standard deviation of three independent experiments. " $\mathrm{P}<0.05$ vs. the CVST group. rhs-TM, recombinant human soluble thrombomodulin; ND, neurological deficit; CVST, cerebral venous sinus thrombosis; TTC, 2,3,5-triphenyltetrazolium chloride.

rhs-TM reduced brain water content. The water content of rat brains was $76.3 \pm 0.15 \%$ in the sham group, $80.3 \pm 0.34 \%$ in the CVST group, and $78.21 \pm 0.24 \%$ in the rhs-TM group. This difference between the CVST and rhs-TM groups was significant $(\mathrm{P}<0.001$; Fig. 2D).

rhs-TM downregulated the $m R N A$ and protein expression levels of HMGB1-RAGE and their downstream effectors in infarcted segments of CVST rat brain. mRNA and protein expression levels of HMGB1-RAGE and their downstream effectors, TNF- $\alpha$, IL- $1 \beta$ and IL- 6 were assessed by RT-qPCR and western blot analysis, respectively. A significant downregulation of HMGB1-RAGE, TNF- $\alpha$, IL-1 $\beta$ and IL-6 mRNA (Fig. 3) and protein (Fig. 4) expression levels was detected in infarcted segments of the brains of rats treated with rhs-TM compared with CVST alone $(\mathrm{P}<0.001)$. In addition, expression levels of apoptosis-associated genes and proteins (caspase-3, Bcl-2 and Bax) in infarcted segments were affected by rhs-TM treatment; however, the differences were not statistically significant when compared with the sham group (caspase- 3 mRNA, $\mathrm{P}=0.052$; Bcl-2 mRNA, $\mathrm{P}=0.192$; Bax mRNA, $\mathrm{P}=0.077$; caspase -3 protein, $\mathrm{P}=0.213$; $\mathrm{Bcl}-2$ protein, $\mathrm{P}=0.06$; $\mathrm{Bax}$ protein, $\mathrm{P}=0.176$ ).

\section{Discussion}

CVST is a rare cerebrovascular disease with various causes. Puerperium, oral hormonal contraception and coagulation disorders remain the most frequently identified risk factors. However, the etiology remains unknown in $~ 15 \%$ of cases $(1,12)$. CVST is associated with protein C deficiency.
Protein $\mathrm{C}$ interacts with the thrombin-TM complex and the endothelial protein $\mathrm{C}$ receptor (EPCR), transforming into activated protein $\mathrm{C}$, which may inactivate factors $\mathrm{Va}$ and VIIIa with protein $\mathrm{S}$ assistance (13). TM is a membrane protein expressed by endothelial cells, including in arteries, veins, capillaries and lymphatic vessels, and in other cell types, including astrocytes, keratinocytes and neutrophils (14). Previous studies verified that TM was part of the anticoagulant protein $\mathrm{C}$ system; however it has become clear that, in addition, TM provides anti-inflammatory protection independently of activated protein $\mathrm{C}$, with or without activation of thrombin-activated fibrinolysis inhibitor (15).

Recombinant TM contains all the extracellular domains (rTMD123, also known as ART-123 or Recomodulin) and has been approved for clinical use in the treatment of disseminated intravascular coagulation in Japan (16). Previous studies have demonstrated that recombinant soluble TM protects against tissue damage and functional deterioration following ischemia in various organs. It may protect against ischemic brain damage via decreasing the expression levels of the proinflammatory cytokines TNF, IL- $1 \beta$ and IL- 6 in the infarcted regions following ischemic stroke (10). Su et al (9) demonstrated Solulin partially restored blood flow following arterial occlusion and reduced ischemia with no overt indications of bleeding experienced with other anticoagulants. Ryang et al (10) demonstrated that Solulin decreased the infarct volume in an artery occlusion model of stroke. In addition, recombinant soluble TM reduced proinflammatory mediators, inhibited macrophage recruitment and suppressed HMGB1-RAGE signaling, to protect against abdominal aortic 

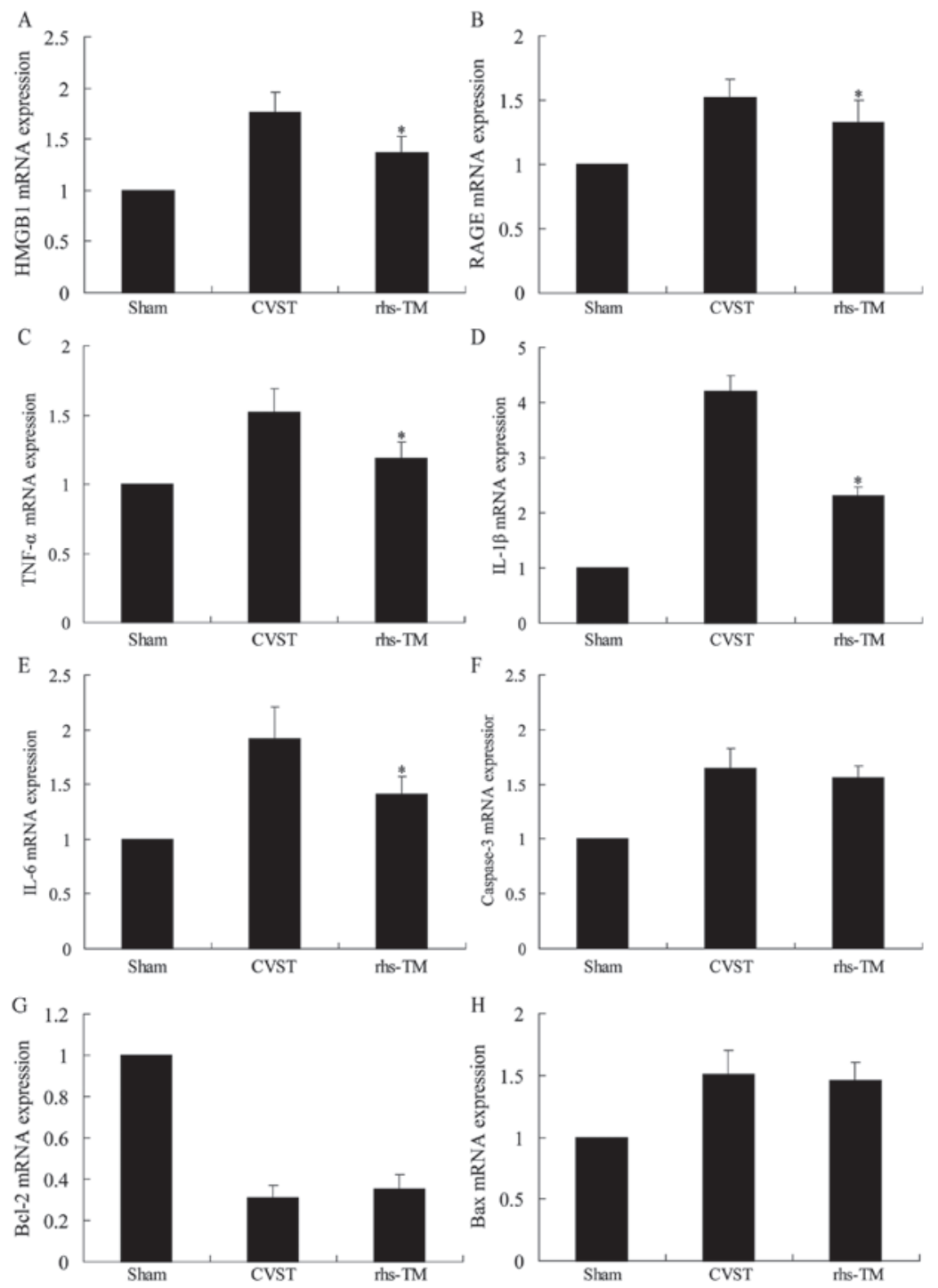

Figure 3. Effect of rhs-TM on mRNA expression levels following CVST. The mRNA expression levels of (A) HMGB1, (B) RAGE, (C) TNF- $\alpha$, (D) IL-1 $\beta$ and (E) IL-6 were significantly downregulated in infarcted segments of the brains of rats treated with rhs-TM compared with CVST alone (P<0.05). The mRNA expression levels of apoptosis-associated $(\mathrm{F})$ caspase-3, $(\mathrm{G}) \mathrm{Bcl}-2$ and $(\mathrm{H}) \mathrm{Bax}$ in infarcted segments of the brains of rats treated with rhs-TM were not significantly different to CVST alone. Data are expressed as the mean \pm standard deviation of three independent experiments. "P<0.05 vs. the CVST alone group. rhs-TM, recombinant human soluble thrombomodulin; CVST, cerebral venous sinus thrombosis; HMGB1, high mobility group box 1; RAGE, receptor for advanced glycation endproducts; TNF- $\alpha$, tumor necrosis factor- $\alpha$; IL, interleukin; Bcl-2, B-cell lymphoma-2; Bax, Bcl-2 associated X.
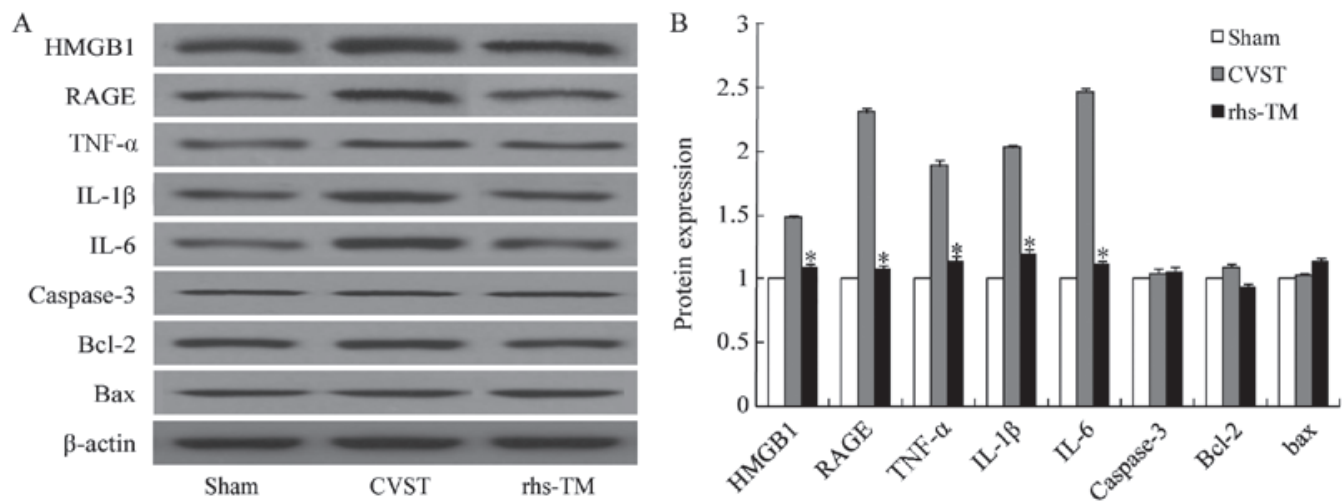

Figure 4. Effect of rhs-TM on protein expression levels following CVST. HMGB1, RAGE, TNF- $\alpha$, IL-1 $\beta$, IL-6, caspase-3, Bcl-2 and Bax protein expression levels were (A) detected and (B) quantified by western blot analysis. The protein expression levels of HMGB1, RAGE, TNF- $\alpha$, IL-1 $\beta$ and IL- 6 were significantly downregulated in infarcted segments of the brains of rats treated with rhs-TM compared with CVST alone $(\mathrm{P}<0.05)$. The protein expression levels of apoptosis-associated caspase-3, Bcl-2 and Bax in infarcted segments of the brains of rats treated with rhs-TM were not significantly different to CVST alone. Data are expressed as the mean \pm standard deviation of three independent experiments. ${ }^{*} \mathrm{P}<0.05$ vs. CVST alone. rhs-TM, recombinant human soluble thrombomodulin; CVST, cerebral venous sinus thrombosis; HMGB1, high mobility group box 1; RAGE, receptor for advanced glycation endproducts; TNF- $\alpha$, tumor necrosis factor- $\alpha$; IL, interleukin; Bcl-2, B-cell lymphoma-2; Bax, Bcl-2 associated X. 
aneurysm development (17). Javanmard et al (18) analyzed the concentrations of soluble TM and soluble EPCR, using ELISA, of 19 CVST patients without protein C or protein S deficiency and 53 healthy controls. The results indicated that the plasma soluble TM level in CVST patients was reduced compared with controls and the adjusted odds ratio for CVST associated with low ( $<10$ th percentile) levels of soluble TM was 2.3 (95\% confidence interval: $1.29-20.08 ; \mathrm{P}=0.012$ ), following adjustment for confounding factors.

HMGB1 is a chromatin-binding protein, which may act as mediator in stroke and other inflammatory diseases $(19,20)$. Previous studies have demonstrated that HMGB1 binding to RAGE and Toll-like receptors contributes to inflammation, affecting the activation of the immune system following stroke $(21,22)$. The lectin-like domain of TM sequesters HMGB1 protein to prevent it from engaging RAGE, which may sustain chronic inflammatory responses and result in tissue damage $(23,24)$. In addition, TM facilitates the proteolytic cleavage of HMGB1 by thrombin (25). In the present study, HMGB1-RAGE mRNA and protein levels were demonstrated to be upregulated in the infarcted segments of rat brains following CVST. rhs-TM administration inhibited neurological deficits and decreased infarction volume, and resulted in a downregulation of the expression levels of HMGB1-RAGE in the penumbra. In addition, the expression levels of the proinflammatory cytokines, TNF- $\alpha$, IL-1 $\beta$ and IL- 6 were decreased. Although a study by Yang et al (26) demonstrated that apoptosis is crucial during CVST development, in the present study rhs-TM administration did not alter the expression levels of the apoptosis-associated caspase-3, Bcl-2 and Bax in the infarcted regions of brains of rats subjected to CVST. These results indicate that the mechanism underlying the protection of the brains of CVST rats by rhs-TM involves the inhibition of inflammation by blocking HMGB1 binding to RAGE, and not the regulation of apoptosis.

In conclusion, although the pathogenesis of CVST remains to be fully elucidated, the results of the present study suggest that the inflammatory response is critical in CVST. rhs-TM reduced infarct volume in a model of CVST, potentially via the inhibition of inflammation. rhs-TM may therefore be a novel potential strategy for the treatment of CVST; however, further studies are required to confirm that rhs-TM does not increase the risk of bleeding in this model.

\section{References}

1. Bousser MG and Ferro JM: Cerebral venous thrombosis: An update. Lancet Neurol 6: 162-170, 2007.

2. Ruiz-Sandoval JL, Chiquete E, Bañuelos-Becerra LJ, Torres-Anguiano C, González-Padilla C, Arauz A, León-Jiménez C, Murillo-Bonilla LM, Villarreal-Careaga J, Barinagarrementería $\mathrm{F}$, et al: Cerebral venous thrombosis in a Mexican multicenter registry of acute cerebrovascular disease: The RENAMEVASC study. J Stroke Cerebrovasc Dis 21: 395-400, 2012

3. Schaller B and Graf R: Cerebral venous infarction: The pathophysiological concept. Cerebrovasc Dis 18: 179-188, 2004.

4. Guenther G and Arauz A; Cerebral venous thrombosis: A diagnostic and treatment update. Neurologia 26: 488-498, 2011.

5. Camerlingo M, Salvi P, Belloni G, Gamba T, Cesana BM and Mamoli A: Intravenous heparin started within the first 3 hours after onset of symptoms as a treatment for acute nonlacunar hemispheric cerebral infarctions. Stroke 36: 2415-2420, 2005.
6. Esmon $\mathrm{CT}$ and Owen WG: Identification of an endothelial cell cofactor for thrombin-catalyzed activation of protein C. Proc Natl Acad Sci USA 78: 2249-2252, 1981.

7. Lane DA, Philippou H and Huntington JA: Directing thrombin. Blood 106: 2605-2612, 2005.

8. Conway EM, Van de Wouwer M, Pollefeyt S, Jurk K, Van Aken H, De Vriese A, Weitz JI, Weiler H, Hellings PW, Schaeffer P, et al: The lectin-like domain of thrombomodulin confers protection from neutrophil-mediated tissue damage by suppressing adhesion molecule expression via nuclear factor kappaB and mitogen-activated protein kinase pathways. J Exp Med 196: 565-577, 2002.

9. Su EJ, Geyer M, Wahl M, Mann K, Ginsburg D, Brohmann H, Petersen KU and Lawrence DA: The thrombomodulin analog Solulin promotes reperfusion and reduces infarct volume in a thrombotic stroke model. J Thromb Haemost 9: 1174-1182, 2011.

10. Ryang YM, Dang J, Kipp M, Petersen KU, Fahlenkamp AV, Gempt J, Wesp D, Rossaint R, Beyer C and Coburn M: Solulin reduces infarct volume and regulates gene-expression in transient middle cerebral artery occlusion in rats. BMC Neurosci 12: 113, 2011.

11. Huang Z, Huang PL, Panahian N, Dalkara T, Fishman MC and Moskowitz MA: Effect of cerebral ischemia in mice deficient in neuronal nitric oxide synthase. Science 265: 1883-1885, 1994

12. Weimar C: Diagnosis and treatment of cerebral venous and sinus thrombosis. Curr Neurol Neurosci Rep 14: 417, 2014.

13. Esmon CT: The protein C pathway. Chest 124 (Suppl 3): 26S-32S, 2003.

14. Wenzel J, Assmann JC and Schwaninger M: Thrombomodulin-a new target for treating stroke at the crossroad of coagulation and inflammation. Curr Med Chem 21: 2025-2034, 2014.

15. Van de WouwerM,PlaisanceS,De Vriese A, WaelkensE,Collen D, Persson J, Daha MR and Conway EM: The lectin-like domain of thrombomodulin interferes with complement activation and protects against arthritis. J Thromb Haemost 4: 1813-1824, 2006.

16. Ito $\mathrm{T}$ and Maruyama I: Thrombomodulin: Protectorate God of the vasculature in thrombosis and infiammation. J Thromb Haemost 9 (Suppl 1): S168-S173, 2011.

17. Lai CH, Shi GY, Lee FT, Kuo CH, Cheng TL, Chang BI, Ma CY, Hsu FC, Yang YJ and Wu HL: Recombinant hman thrombomodulin suppresses experimental abdominal aortic aneurysms induced by calcium chloride in mice. Ann Surg 258: 1103-1110, 2013.

18. Javanmard SH, Shahsavarzadeh T and Saadatnia M: Soluble thrombomodulin and endothelial cell protein $\mathrm{C}$ receptor levels in patients with cerebral venous and sinus thrombosis. Eur Neurol 70: 156-158, 2013.

19. Scaffidi P, Misteli T and Bianchi ME: Release of chromatin protein HMGB1 by necrotic cells triggers inflammation. Nature 418: 191-195, 2002.

20. Andrassy M, Volz HC, Igwe JC, Funke B, Eichberger SN, Kaya Z, Buss S, Autschbach F, Pleger ST, Lukic IK, et al: High-mobility group box-1 in ischemia-reperfusion injury of the heart. Circulation 117: 3216-3226, 2008.

21. Abeyama K, Stern DM, Ito Y, Kawahara K, Yoshimoto Y, Tanaka M, Uchimura T, Ida N, Yamazaki Y, Yamada S, et al: The $\mathrm{N}$-terminal domain of thrombomodulin sequesters high-mobility group-B1 protein, a novel antiinflammatory mechanism. J Clin Invest 115: 1267-1274, 2005.

22. Tsung A, Klune JR, Zhang X, Jeyabalan G, Cao Z, Peng X, Stolz DB, Geller DA, Rosengart MR and Billiar TR: HMGB1 release induced by liver ischemia involves Toll-like receptor 4 dependent reactive oxygen species production and calcium-mediated signaling. J Exp Med 204: 2913-2923, 2007.

23. Rauvala H and Rouhiainen A: Physiological and pathophysiological outcomes of the interactions of HMGB1 with cell surface receptors. Biochim Biophys Acta 1799: 164-170, 2010.

24. Schmidt AM, Yan SD, Yan SF and Stern DM: The multiligand receptor RAGE as a progression factor amplifying immune and inflammatory responses. J Clin Invest 108: 949-955, 2001.

25. Ito T, Kawahara K, Okamoto K, Yamada S, Yasuda M, Imaizumi H, Nawa Y, Meng X, Shrestha B, Hashiguchi T and Maruyama I: Proteolytic cleavage of high mobility group box 1 protein by thrombin-thrombomodulin complexes. Arterioscler Thromb Vasc Biol 28: 1825-1830, 2008.

26. Yang H, Meng Z, Zhang C, Zhang P and Wang Q: Establishing a new rat model of central venous sinus thrombosis and analyzing its pathophysiological and apoptotic changes. J Neurosci Methods 203: 130-135, 2012. 